\title{
A Retrospective Cohort Study Evaluates Clinical Value of Anlotinib in Persistent, Metastatic, or Recurrent Cervical Cancer After Failure of First-Line Therapy
}

\author{
Hui Yang $\mathbb{1}^{1-3}$ \\ Shaoxing Sun $\mathbb{1}^{1-3}$ \\ Zijie $\mathrm{Mei}^{\mathrm{I}-3}$ \\ Qingming Xiang ${ }^{1-3}$ \\ Chunxu Yang ${ }^{1-3}$ \\ Min Chen ${ }^{1-3}$ \\ Conghua Xie $\mathbb{D}^{1-3}$ \\ Yunfeng Zhou (iD) ${ }^{1-3}$ \\ Hui Qiu ${ }^{1-3}$
}

'Department of Radiation and Medical Oncology, Zhongnan Hospital of Wuhan University, Wuhan, People's Republic of China; ${ }^{2}$ Hubei Key Laboratory of Tumor Biological Behaviors, Zhongnan Hospital of Wuhan University, Wuhan, People's Republic of China; ${ }^{3}$ Hubei Cancer Clinical Study Center, Zhongnan Hospital of Wuhan University, Wuhan, People's Republic of China
Background: Anlotinib is an oral anti-angiogenesis inhibitor targeting vascular endothelial growth factor receptors (VEGFRs), platelet-derived growth factor receptors, fibroblast growth factor receptors, etc., and its clinical value in cervical cancer is rarely reported. We designed a retrospective study to evaluate the efficacy and safety of anlotinib in patients with persistent, metastatic, or recurrent cervical cancer who have failed first-line therapy, and compare the efficacy of anlotinib with that of apatinib which targets only VEGFR2 and has shown efficacy in recent studies.

Methods: Fifty-two patients with persistent, metastatic, or recurrent cervical cancer who failed first-line therapy and administrated anlotinib or apatinib as monotherapy or combination with chemo-, radio- or immunotherapy were included in this study. Among the 52 patients, 20 patients who received anlotinib from January 2019 to August 2020 were defined as anlotinib group, whereas 32 patients who received apatinib from our previous study were selected as apatinib group. The safety, objective response rate (ORR), disease control rate (DCR), progression-free survival (PFS), and overall survival (OS) were reviewed and recorded.

Results: The ORR and DCR in patients receiving anlotinib were $25 \%$ and $80 \%$, respectively. The median PFS and OS in anlotinib group were significantly longer than those in apatinib group, respectively (PFS: 5 months vs 3 months, $\mathrm{p}=0.015$; OS: 10 months vs 5 months, $\mathrm{p}=0.008$ ). Moreover, the patients treated with anlotinib had better survival with a significantly lower cumulative incidence of cancer-related death than those treated with apatinib ( $\mathrm{HR}=0.31,95 \%$ CI: $0.13-0.77, \mathrm{p}=0.012$ ). The most common adverse effects in the patients treated with anlotinib were hypertension (20\%), fatigue (20\%), and nausea (15\%). No drug-related death occurred.

Conclusion: Anlotinib showed beneficial efficacy and safety and can be a treatment option for patients with persistent, metastatic, or recurrent cervical cancer who have failed the firstline therapy.

Keywords: angiogenesis, anlotinib, cervical cancer, efficacy, safety, retrospective study

\section{Introduction}

Cervical cancer is still the most common cancer in the female reproductive system. ${ }^{1}$ After standardized treatment, the average 5-year overall survival (OS) of patients with stage IIII cervical cancer is $45 \%$. However, the 5 -year OS is only $15 \%$ after the disease becomes persistent, recurrent, or metastatic due to ineffective strategies. ${ }^{2}$ Therefore, it is urgent to explore effective treatment strategies for persistent, recurrent, or metastatic cervical cancer. 
Anti-angiogenesis targeted therapy has become one of the treatment strategies for many tumors. Cervical cancer is no exception. The GOG240 study showed that compared to paclitaxel plus cisplatin alone, paclitaxel and cisplatin combined with bevacizumab, which targeted vascular endothelial growth factor (VEGF), can improve the response rates (RR) (from $36 \%$ to $48 \%$ ), progression-free survival (PFS) (from 5.9 to 8.2 months) and OS (from 13.3 to 17 months). ${ }^{3}$ Similarly, other anti-angiogenesis drugs, apatinib, cediranib, and pazopanib, have shown effective and low toxicity in advanced and recurrent cervical cancer. ${ }^{4}$ However, recent studies have found that when tumor tissue is hypoxic, it can produce three signal molecules: VEGF, platelet-derived growth factor (PDGF), and fibroblast growth factor (FGF). When VEGF-related signaling pathways are blocked, tumor tissues can up-regulate the expression of other cytokines through escape and bypass activation (such as FGF and PDGF) to maintain their nutritional supply. ${ }^{5}$ It suggests that simultaneous blocking of the downstream signal pathways mediated by VEGF-receptor (VEGFR), FGF-receptor (FGFR), and PDGF-receptor (PDGFR) could more comprehensively inhibit tumor angiogenesis, thereby exerting a favorable antitumor effect.

Anlotinib is an anti-angiogenic drug that can fully inhibit VEGFR, PDGFR, and FGFR. It can inhibit tumor cell proliferation and migration by inhibiting stem cell factor receptor (c-Kit) and cellular-mesenchymal epithelial transition factor (c-Met). ${ }^{6}$ Due to the unique cyclopropyl structure of the drug, the half-maximal inhibitory concentration (IC50) of anlotinib at each target is low, and the activity is vigorous; more importantly, the main target of anlotinib is tyrosine kinase (TK). ${ }^{7}$ These characteristics enable anlotinib to inhibit angiogenesis and tumor growth simultaneously, and it is more delayed than other singletarget anti-angiogenic drugs to develop drug resistance and has fewer side effects. It has been approved by the China State Food and Drug Administration (CSFDA) for locally advanced or metastatic non-small cell lung cancer patients who have progressed or relapsed after receiving at least two types of systemic chemotherapy in the past. ${ }^{8}$ Moreover, studies have reported that the drug has shown promising efficacy and safety in esophageal squamous cell carcinoma, ${ }^{9}$ soft tissue sarcoma, ${ }^{10}$ medullary thyroid carcinoma, ${ }^{11}$ ovarian cancer, ${ }^{12}$ and other tumors. ${ }^{13}$ However, no clinical studies have reported the efficacy and safety of anlotinib in patients with cervical cancer, let alone assessing whether the efficacy of anlotinib is better than single anti-VEGF drugs.

Apatinib is an oral anti-angiogenesis drug that only targets VEGFR2. Recently, others and our previous studies have shown that apatinib has efficacy and safety in patients with cervical cancer. ${ }^{4,14-20}$ To explore the efficacy of anlotinib in persistent, recurrent, or metastatic cervical cancer and determine whether the efficacy of anlotinib is superior to single anti-VEGF drugs, we selected patients who received apatinib in our previous studies as a control group. Therefore, this study performed a retrospective study not only to evaluate the efficacy and safety of anlotinib in persistent, recurrent, or metastatic cervical cancer, but also to compare the difference in overall response rate (ORR), disease control rate (DCR), PFS, and OS between anlotinib group and apatinib group.

\section{Materials and Methods}

\section{Patient Eligibility}

This retrospective study was performed to analyze clinical data on patients with persistent, metastatic, or recurrent cervical cancer who were treated with anlotinib or apatinib and met the inclusion and exclusion criteria in Zhongnan Hospital of Wuhan University.

All patients in this study had undergone one comprehensive treatment and signed informed consent to take anlotinib or apatinib. Persistent and recurrent cervical cancer that failed the first-line therapy was defined as disease progression following initial surgery, chemo-, or radiotherapy in or after 6 months, respectively, and then the disease progressed again after receiving systemic treatment. Metastatic cervical cancer that failed the first-line therapy was defined as the disease progression that appeared after systemic therapy in the patients with stage IVB (according to the International Federation of Gynecology and Obstetrics (FIGO) staging) cervical cancer. Patients had at least one measurable lesion and an Eastern Cooperative Oncology Group (ECOG) performance status score of 0,1 , or 2 . Before treatment, patients were confirmed not to be treated with antiangiogenesis drugs and no risk of bleeding and/or have grade II coronary heart disease, uncontrollable high blood pressure, arrhythmia, cardiac insufficiency, nausea and swallow, chronic diarrhea, and intestinal obstruction. Patients who have not finished at least one cycle of anlotinib or apatinib treatment were also excluded from this study. The patients in anlotinib group were included in our hospital from January 2019 to August 2020. The patients in apatinib 
group were selected from our previous retrospective cohort study. $^{14}$

\section{Treatment and Dose Modification}

Anlotinib was initially taken $12 \mathrm{mg}$ orally every day for 14 days and then stopped for 7 days ( 21 days as a cycle). The daily dosage was reduced from $12 \mathrm{mg}$ to $10 \mathrm{mg}$ when the patients could not tolerate it. If the patients were still intolerant, the daily dosage was adjusted from $10 \mathrm{mg}$ to $8 \mathrm{mg}$. After radiotherapy, patients suspended anlotinib treatment. After chemotherapy, patients were continually administered anlotinib till disease progression or unacceptable toxicity. The treatment and dose modification of apatinib were described in our previous study. ${ }^{14}$

\section{Efficacy and Safety Assessments}

All patients in anlotinib group were followed up until June 2021. According to the solid tumor efficacy evaluation standard (RECIST 1.1), tumor response included complete response $(\mathrm{CR})$, partial response (PR), stable disease (SD), and progressive disease (PD). Overall response rate (ORR) was calculated by the ratio of CR plus $\mathrm{PR}(\mathrm{CR}+\mathrm{PR})$ to the total number of patients. Disease control rate (DCR) was calculated by the percentage of $\mathrm{CR}, \mathrm{PR}$ plus $\mathrm{SD}(\mathrm{CR}+\mathrm{PR}+\mathrm{SD})$ to the total number of patients. Progression-free survival (PFS) was defined from initiating anlotinib treatment to clinical or radiographic progression or death. Overall survival (OS) was measured from firstly anlotinib therapy to death or last contact. Based on the National Cancer Institute Common Terminology Criteria for Adverse Events, version 5.0 (CTCAE 5.0), the toxicity was recorded from patients' medical history, telephone follow-up, or laboratory examination results. The records of efficacy in patients treated with apatinib were described in our previous study. ${ }^{14}$

\section{Statistical Analysis}

Statistical analysis was performed using SPSS version 25 (IBM, NC, USA). Median (95\% confidence interval) or the number of patients (percentage) were used to describe patients' survival and clinical characteristics. The Wilcoxon or Kruskal-Wallis $H$-test was applied to analyze the efficacy difference between two or three groups. Kaplan-Meier method and the Log rank test were used to compare the survival of patients between different treatment groups. The multivariate Cox regression analysis was used to evaluate the independent prognostic factors. The difference was defined as statistically significant when $\mathrm{p}<0.05$ (two-tails).

\section{Results}

\section{Patient Characteristics}

A total of 52 patients with persistent, recurrent, or metastatic cervical cancer who had failed first-line therapy were included in this retrospective study. Among the 52 patients, 20 patients who received anlotinib from January 2019 to August 2020 were defined as anlotinib group, whereas 32 patients who received apatinib from our previous study were selected as apatinib group. All patients in this study have previously received surgery and/or chemotherapy and radiotherapy; no bevacizumab was received before. In anlotinib group, $80 \%$ of patients experienced disease progression, and $70 \%$ of patients died. The baseline characteristics were summarized in Table 1. There were no differences between anlotinib and apatinib groups among patients in different age groups $(p=0.855)$, different administration strategies $(p=0.072)$, different initial conditions $(\mathrm{p}=1.000)$, and different prior treatments $(p=1.000)$. But the patients in different treatment lines $(p=0.034)$ and pathological types $(p=0.033)$ showed differences between the anlotinib and apatinib groups.

Table I The Baseline Characteristics in This Study

\begin{tabular}{|l|l|l|l|}
\hline Characteristics & $\begin{array}{l}\text { Anlobinib } \\
\mathbf{n = 2 0 ( \% )}\end{array}$ & $\begin{array}{l}\text { Apatinib } \\
\mathbf{n = 3 2 ( \% )}\end{array}$ & p-value \\
\hline Age & $7(35 \%)$ & $12(37.5 \%)$ & 0.855 \\
$\geq 50$ & $13(65 \%)$ & $20(62.5 \%)$ & \\
\hline Pathological types & & & \\
SCC & $13(65 \%)$ & $29(90.6 \%)$ & $\mathbf{0 . 0 3 3}$ \\
AC and others & $7(35 \%)$ & $3(9.4 \%)$ & \\
\hline Treatment lines & & & \\
Second & $6(30 \%)$ & $18(56.3 \%)$ & $\mathbf{0 . 0 3 4}$ \\
Third & $9(45 \%)$ & $13(40.5 \%)$ & \\
Further & $5(25 \%)$ & $1(3.1 \%)$ & \\
\hline Administration strategies & & & \\
Monotherapy & $4(20 \%)$ & $19(59.4 \%)$ & 0.072 \\
Combined with chemotherapy & $9(45 \%)$ & $9(28.1 \%)$ & \\
Combined with radiotherapy & $2(10 \%)$ & $4(12.5 \%)$ & \\
Combined with immunotherapy & $5(25 \%)$ & $0(0.0 \%)$ & \\
\hline Initial conditions & & & \\
Recurrent & $18(90 \%)$ & $29(90.6 \%)$ & 1.000 \\
Metastatic & $2(10 \%)$ & $3(9.4 \%)$ & \\
\hline Prior treatments & & & \\
Surgery, radiotherapy, and chemotherapy & $11(55 \%)$ & $18(56.3 \%)$ & 1.000 \\
Radiotherapy and chemotherapy & $9(45 \%)$ & $14(43.7 \%)$ & \\
\hline Notes The bold value indion & \\
\hline
\end{tabular}

Notes: The bold value indicates $\mathrm{p}$-value is statistically significant.

Abbreviations: SCC, squamous cervical carcinoma; AC, cervical adenocarcinoma. 


\section{Efficacy}

In anlotinib group, at the end of follow-up time on June 30, 2021, no patient occurred CR, 5 patients showed PR, 11 patients had SD, 4 patients had PD. The ORR and DCR were $25 \%$ and $80 \%$, respectively (Table 2 ). There was no statistical difference in the efficacy of anlotinib among patients of different age groups ( $\mathrm{p}=0.157)$, different pathological types $(\mathrm{p}=0.877)$, different treatment lines of anlotinib $(\mathrm{p}=0.402)$, different anlotinib administration strategies $(\mathrm{p}=0.610)$, and different initial conditions $(\mathrm{p}=0.290)$ (Table 3). In apatinib group, no patient occurred CR, 7 patients showed PR, 17 patients had SD, 8 patients had PD. The ORR and DCR were $21.88 \%$ and $75 \%$, respectively (Table 2). When we compared the tumor response between anlotinib and apatinib groups, no statistically significant had been found $(\mathrm{p}=0.678)$ (Table 2$)$. These results suggested that similar to apatinib, the anlotinib was also showed efficacy in patients with cervical cancer.

\section{Survival Analysis}

In anlotinib group, the median PFS and OS were 5.0 months (95\% CI: $0-10.48)$ and 10.0 months (95\% CI: 6.10-13.90), respectively (Table 4 and Figure 1). The 1 -year survival rate was $43.7 \%$. There were no differences of PFS and OS in patients of different age groups (PFS: $\mathrm{p}=0.749$, OS: $\mathrm{p}=0.976$ ), different pathological types (PFS: $\mathrm{p}=0.299$, OS: $\mathrm{p}=0.179$ ), different treatment lines of anlotinib (PFS: $p=0.286$, OS: $p=0.177$ ), and different initial conditions (PFS: $\mathrm{p}=0.687$, OS: $\mathrm{p}=0.586$ ), respectively (Table 4). But the differences of PFS and OS in patients with different anlotinib administration strategies were statistically significant (PFS: $\mathrm{p}=0.0001$, OS: $\mathrm{p}=0.030$ ) (Table 4). There were only 2 patients administrated anlotinib combined with radiotherapy. The overall survival

Table 2 The Difference of Tumor Response Between Anlotinb and Apatinib

\begin{tabular}{|l|r|r|r|}
\hline Tumor Response & Anlotinib & Apatinib & p-value \\
\hline CR & 0 & 0 & \\
PR & 5 & 7 & \\
SD & $1 \mathrm{r}$ & 17 & \\
PD & 4 & 8 & 0.678 \\
$\mathrm{ORR}^{\mathrm{a}}(\%)$ & $25 \%$ & $21.88 \%$ & \\
$\mathrm{DCR}^{\mathrm{b}}(\%)$ & $80 \%$ & $75 \%$ & \\
\hline
\end{tabular}

Notes: ${ }^{a} O R R$, the ratio of the number of $C R$ and PR (CR+PR) patients to the total number of patients; ${ }^{b} D C R$, the ratio of the number of $C R, P R$, and $S D(C R+P R+S D)$ patients to the total number of patients.

Abbreviations: $\mathrm{CR}$, complete response; $\mathrm{PR}$, partial response; SD, stable disease; $\mathrm{PD}$, progressive disease; $\mathrm{DCR}$, disease control rate; ORR, objective response rate.
Table 3 The Difference of Tumor Response Between Different Characteristics in Anlotinib Group

\begin{tabular}{|l|c|c|c|c|c|}
\hline Characteristics & CR & PR & SD & PD & p-value \\
\hline Total & 0 & 5 & II & 4 & \\
\hline Age & & & & & \\
$<50$ & 0 & 1 & 3 & 3 & 0.157 \\
$\geq 50$ & 0 & 4 & 8 & I & \\
\hline Pathological types & & & & & \\
SCC & 0 & 3 & 8 & 2 & 0.877 \\
AC and others & 0 & 2 & 3 & 2 & \\
\hline Treatment lines & & & & & \\
Second & 0 & 3 & 2 & 1 & 0.402 \\
Third & 0 & 2 & 5 & 2 & \\
Further & 0 & 0 & 4 & I & \\
\hline Administration strategies & & & & & \\
Monotherapy & 0 & I & 2 & I & 0.610 \\
Combined with chemotherapy & 0 & I & 6 & 2 & \\
Combined with radiotherapy & 0 & I & 0 & I & \\
Combined with immunotherapy & 0 & 2 & 3 & 0 & \\
\hline Initial conditions & & & & & \\
Recurrent & 0 & 5 & 9 & 3 & 0.290 \\
Metastatic & 0 & 0 & 2 & 1 & \\
\hline
\end{tabular}

Abbreviations: CR, complete response; PR, partial response; SD, stable disease; $\mathrm{PD}$, progressive disease; SCC, squamous cervical carcinoma; AC, cervical adenocarcinoma.

time for them was 4.5 and 6.0 months, respectively. Since only 1 of 5 patients in the group of anlotinib combined with immunotherapy have died, the median OS had not reached yet. The overall survival of the patient who was died in the group of anlotinib combined with immunotherapy was 8 months. At the end of follow-up time, the other 4 patients in the group of anlotinib combined with immunotherapy were still alive, and their OS was 11, 12, 12 and 20 months, respectively. As shown in Figure 2, the PFS and OS rate seemed the highest in patients administrated anlotinib combined with immunotherapy than those in other administration strategies. In contrast, the PFS and OS rate was the lowest in patients administrated anlotinib combined with radiotherapy. However, the association between different anlotinib administration strategies and prognosis in this study cannot be elucidated because of the small sample size and short follow-up time.

When we performed the Kaplan-Meier method and Log rank test to analyze the difference of PFS and OS between patients treated with anlotinib and apatinib, the results revealed that the median PFS and OS of patients treated with apatinib were 3 months (95\% CI: $1.42-4.58$ months) 
Table 4 Univariable Analysis of PFS and OS in Anlotinib Group

\begin{tabular}{|c|c|c|c|c|}
\hline Characteristics & mPFS in Months $(95 \% \mathrm{Cl})$ & p-value & mOS in Months $(95 \% \mathrm{Cl})$ & p-value \\
\hline Total & $5.0(0-10.48)$ & & $10(6.10-13.90)$ & \\
\hline \multicolumn{5}{|l|}{ Age } \\
\hline$<50$ & $3.5(0-7.35)$ & 0.749 & $10(0-20.27)$ & 0.976 \\
\hline$\geq 50$ & $6.5(2.39-10.61)$ & & II.5(5.68-17.32) & \\
\hline \multicolumn{5}{|l|}{ Pathological types } \\
\hline SCC & $7.5(0-16.31)$ & 0.299 & $14(1 \mid .28-16.72)$ & 0.179 \\
\hline$A C$ and others & $3.5(0-9.92)$ & & $8(5.66-10.34)$ & \\
\hline \multicolumn{5}{|l|}{ Line of anlotinib } \\
\hline Second & $12.75(0-25.85)$ & 0.286 & $14(2.55-25.45)$ & 0.177 \\
\hline Third & $3.5(1.31-5.70)$ & & $8(2.89-13.11)$ & \\
\hline Further & $7.5(0-16.09)$ & & $10(4.63-15.37)$ & \\
\hline \multicolumn{5}{|l|}{ Administration strategies } \\
\hline Monotherapy & $7.5(0-17.50)$ & 0.0001 & II.5(0.23-22.77) & 0.030 \\
\hline Combined with chemotherapy & $3.5(2.04-4.96)$ & & $9.5(5.12-13.88)$ & \\
\hline Combined with radiotherapy & 0.5 & & 4.5 & \\
\hline Combined with immunotherapy & Not reached & & Not reached & \\
\hline \multicolumn{5}{|l|}{ Initial conditions } \\
\hline Recurrent & $6.5(1.12-11.88)$ & 0.687 & II.5(8.45-14.56) & 0.586 \\
\hline Metastatic & $5(0-12.20)$ & & $8(4.80-11.20)$ & \\
\hline
\end{tabular}

Note: The bold value indicates $\mathrm{p}$-value is statistically significant.

Abbreviations: mPFS, median progression-free survival; mOS, median overall survival; SCC, squamous cervical carcinoma; AC, cervical adenocarcinoma.

and 5 months (95\% CI: 3.49-6.51 months), respectively, which were significantly shorter than those of patients treated with anlotinib (PFS, $\mathrm{p}=0.015$; OS, $\mathrm{p}=0.008$ ) (Figure 3). The multivariate analysis showed that treated with anlotinib was an independent protecting factor only in $\mathrm{OS}(\mathrm{HR}=0.31$, 95\% CI 0.13-0.77, $\mathrm{p}=0.012$ ), not in PFS (HR $=0.47,95 \% \mathrm{CI}$
0.21-1.09, $\mathrm{p}=0.080$ ) (Figure 4). In addition, third-line treatment with anlotinib or apatinib was an independent risk factor for both PFS $(\mathrm{HR}=2.04,95 \%$ CI $1.02-4.07$, $\mathrm{p}=0.044)$ and $\mathrm{OS}(\mathrm{HR}=2.16,95 \%$ CI 1.01-4.63, $\mathrm{p}=0.047)$ (Figure 4). These results showed that the efficacy of anlotinib may be superior to that of apatinib.
A

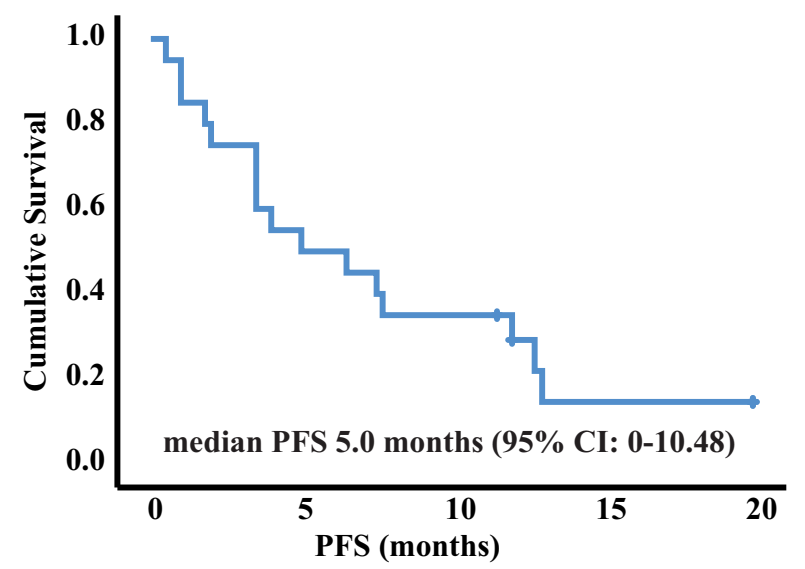

B

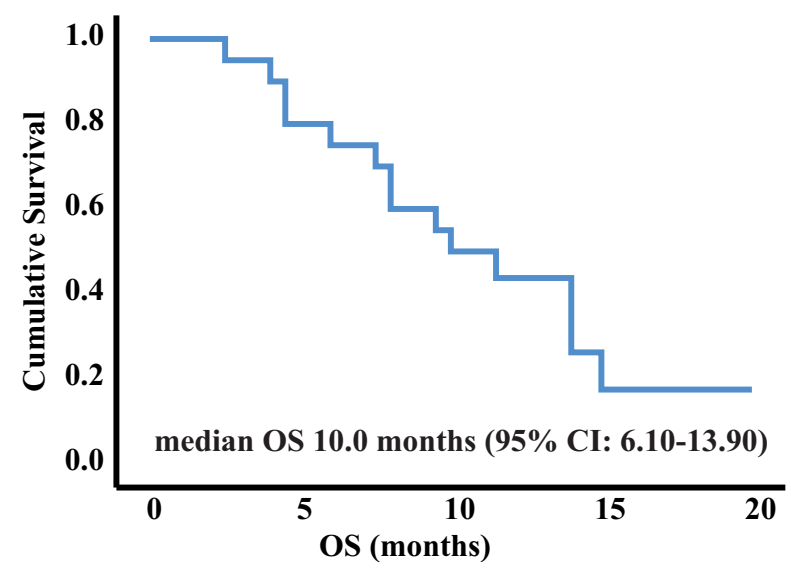

Figure I The Kaplan-Meier analysis and Log rank test assess the PFS (A) and OS (B) of patients in anlotinib group. Abbreviations: PFS, progression-free survival; OS, overall survival. 

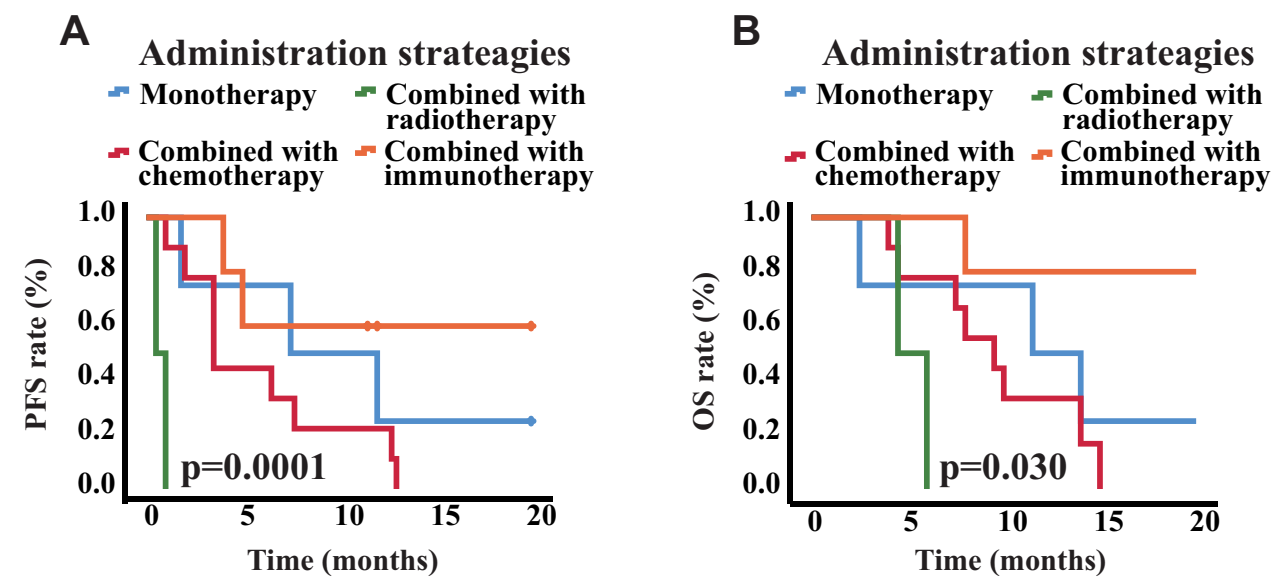

Figure 2 (A) The survival curve of PFS in patients after administrated anlotinib with different strategies. (B) The survival curve of OS in patients after administrated anlotinib with different strategies.

Abbreviations: PFS, progression-free survival; OS, overall survival.

A

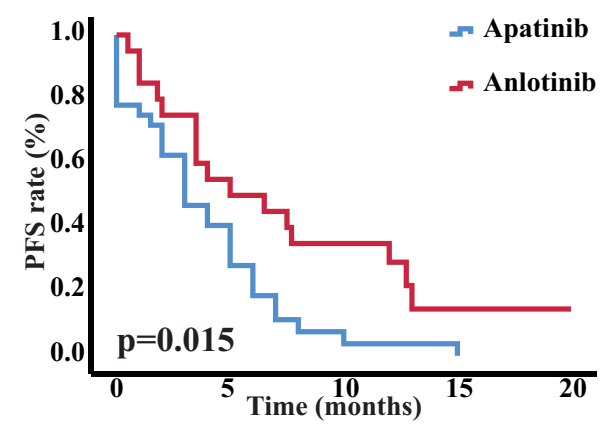

B

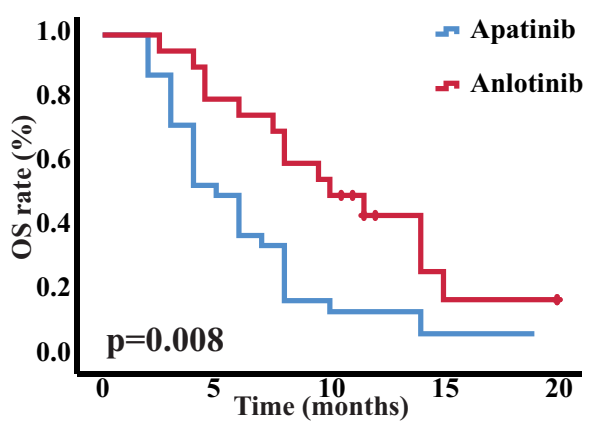

Figure 3 The Kaplan-Meier analysis and Log rank test assess the difference of PFS (A) and OS (B) between the patients treated with anlotinib and those treated with apatinib.

Abbreviations: PFS, progression-free survival; OS, overall survival.

Anlotinib vs. Apatinib

Age $(=\mathbf{5 0}$ vs. $<\mathbf{5 0})$

Pathological type (AC and others vs. SCC)

Treatment lines

Third vs. Second

Further vs. Second

Administration strategies

Combined with chemotherapy vs. Monotherapy

Combined with radiotherapy vs. Monotherapy

Combined with immunotherapy vs. Monotherapy

Initial condition (Metastatic vs. Recurrent)
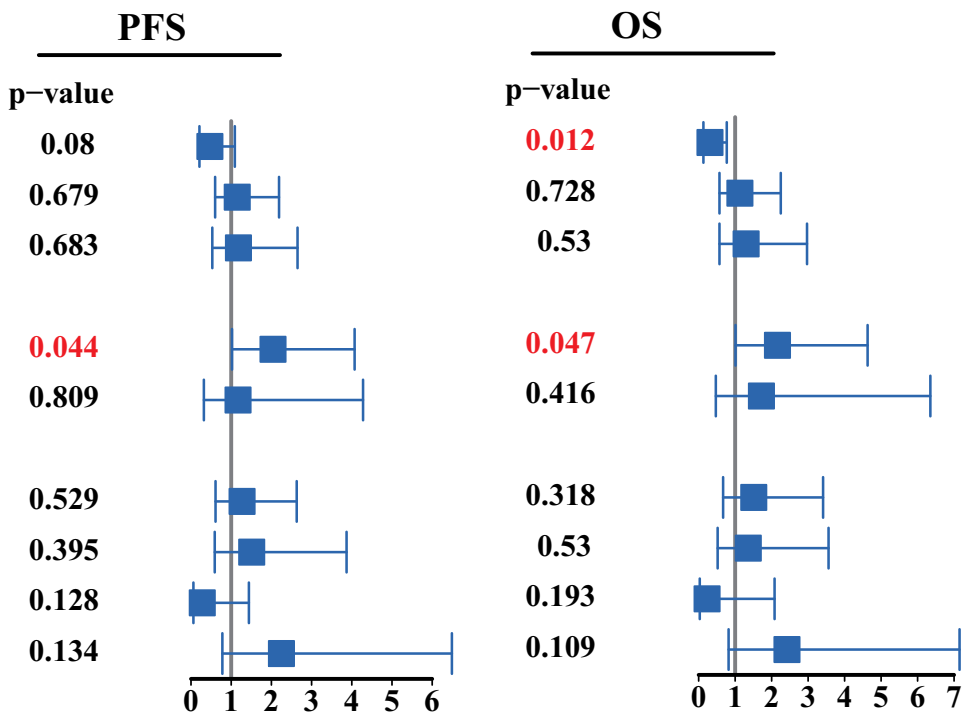

$<-$ Protect Factor- - Risk Factor $->\quad<-$ Protect Factor - -Risk Factor $->$

Figure 4 The multivariate analysis of PFS and OS in this study.

Abbreviations: PFS, progression-free survival; OS, overall survival; SCC, squamous cervical carcinoma; AC, cervical adenocarcinoma. 


\section{Adverse Effects (AEs)}

In this study, 11 people experienced adverse action, including 2 with the hand-foot syndrome, 3 with nausea, 4 with hypertension, 4 with fatigue, 2 with neutropenia, 1 with proteinuria, 1 with vomiting, 1 with mucositis oral, and 1 with diarrhea (Table 5). Two patients had Grade 3 adverse events of hypertension, and their blood pressure could be controlled after taking antihypertensive drugs. One patient had grade 3 neutropenia. After stopping anlotinib and receiving granulocyte colony-stimulating factor, it can be restored to the normal level. When received anlotinib again, the dose will be reduced from $12 \mathrm{mg}$ to $10 \mathrm{mg}$. One patient developed severe fatigue and mucositis oral, respectively, which resolved after stopping anlotinib. The total incidence of grade 3-4 adverse events was $30 \%$. The remaining toxicities were grade 1 to 2 .

\section{Discussion}

In this study, we demonstrate for the first time that anlotinib showed efficacy and safety in patients with persistent, metastatic, or recurrent cervical cancer who have failed the first-line therapy. Furthermore, this study revealed that compared the efficacy of patients treated with anlotinib to the patients treated with apatinib, the patients treated with anlotinib was an independent protecting factor in OS.

In 1971, Professor Judah Folkman published a new theory that "tumor growth depends on angiogenesis" and proposed an "anti-angiogenesis" tumor treatment strategy. ${ }^{21}$ Since then, tumor-targeted anti-angiogenesis therapy has become one of the treatment methods for many tumors. In cervical cancer, the results of GOG240 make bevacizumab combined with paclitaxel and cisplatin become the standard first-line treatment for recurrent and metastatic cervical cancer. However, due to the lack of effective treatment methods, the National Comprehensive Cancer Network (NCCN) guidelines recommend that patients with recurrent and metastatic cervical cancer who have failed first-line treatment can participate in clinical trials to seek new effective remedies, which is essential to improve the overall survival rate of cervical cancer. Studies have shown that alternative angiogenic pathway activated was one of the reasons for anti-VEGF resistance. ${ }^{5}$ The alternative angiogenic pathway includes FGF, PDGF, angiopoietin 1, etc. ${ }^{22}$ Blocking VEGF, FGF and PDGF at the same time may more comprehensively inhibit tumor angiogenesis than just anti-VEGF, thereby exerting an antitumor effect.

Some drugs targets both VEGF, FGF, and PDGF have been studied in cervical cancer. ${ }^{23}$ Pazopanib targets the VEGFR, PDGFR, FGFR, and c-Kit. Studies have explored the efficacy of pazopanib monotherapy in recurrent and metastatic cervical cancer. ${ }^{24}$ The results showed that the ORR, DCR, median PFS, and OS of pazopanib were $9.5 \%$, $52.7 \%, 4.5$, and 12.4 months, respectively. ${ }^{24}$ Sunitinib, similar to pazopanib, could target VEGFR, PDGFR, and c-Kit. ${ }^{25}$ But studies showed that sunitinib is highly toxic without any survival benefit in both PFS and OS. ${ }^{25}$ In this study, anlotinib was used to treat recurrent metastatic cervical cancer that failed the first-line treatment. The ORR and DCR were $25 \%$ and $80 \%$, respectively, which were higher than the results of pazopanib. These may be

Table 5 Adverse Effects (AEs) in Anlotinib Group

\begin{tabular}{|c|c|c|c|c|c|}
\hline \multirow[t]{2}{*}{ Adverse Event } & \multirow[t]{2}{*}{ Total n (\%) } & \multicolumn{4}{|c|}{ No. of Patients } \\
\hline & & Grade I & Grade 2 & Grade 3 & Grade 4 \\
\hline Hand-foot syndrome & $2(10 \%)$ & 2 & 0 & 0 & 0 \\
\hline Nausea & $3(15 \%)$ & 3 & 0 & 0 & 0 \\
\hline Hypertension & $4(20 \%)$ & 0 & 2 & 2 & 0 \\
\hline Fatigue & $4(20 \%)$ & 3 & 0 & I & 0 \\
\hline Hemorrhage & $0(0 \%)$ & 0 & 0 & 0 & 0 \\
\hline Neutropenia & $2(10 \%)$ & I & 0 & I & 0 \\
\hline Canker sore & $0(0 \%)$ & 0 & 0 & 0 & 0 \\
\hline Proteinuria & $\mathrm{I}(5 \%)$ & 0 & 0 & I & 0 \\
\hline Vomiting & $I(5 \%)$ & I & 0 & 0 & 0 \\
\hline Mucositis oral & $I(5 \%)$ & 0 & 0 & I & 0 \\
\hline Diarrhea & $I(5 \%)$ & I & 0 & 0 & 0 \\
\hline None & $9(45 \%)$ & 0 & 0 & 0 & 0 \\
\hline
\end{tabular}


because anlotinib anlotinib targets not just VEGFR, PDGFR, FGFR, and c-Kit, but also c-Met. Some studies have found that c-Met can be activated through negative feedback exerted by VEGF. ${ }^{26}$ So inhibiting c-Met is a method to overcome resistance to anti-VEGF therapy. ${ }^{5}$ However, the median PFS and OS in this study were 5 and 10 months, respectively, which were identical to those in the pazopanib study. Without treatment, the 1-year survival rate for patients with persistent, metastatic, or recurrent cervical cancer is less than $20 \% .{ }^{27}$ In this study, the 1 -year survival rate was $43.7 \%$, indicating that anlotinib may have efficacy in patients with cervical cancer.

In 2020, Xu et al have reported results from a Phase II study of sintilimab plus anlotinib for patients with persistent, recurrent, or metastatic cervical cancer in ESMO ${ }^{28}$ The ORR and DCR were $70.6 \%$ and $100 \%$, respectively. The median PFS had not reached. Similarly, the ORR and DCR of patients who were treated anlotinib combined with immunotherapy in our study were $40 \%$ and $100 \%$, respectively. Although the median OS of patients treated with anlotinib and immunotherapy had not reached, the survival curve in Figure 1B demonstrated that the patients treated with anlotinib and immunotherapy live a relatively longer life than others. Anlotinib has been shown in several studies to alleviate immunosuppression by enhancing T cell, NK cell, and APC cell infiltration and reducing the formation and aggregation of immunosuppressive Treg cells and TAM cells. ${ }^{29,30}$ Thus, anlotinib combined with immunotherapy might be an effective therapy in cervical cancer.

When we compared the efficacy of anlotinib in this study to that of apatinib reported in our previous study, ${ }^{14}$ the results showed that the patients treated with anlotinib had a relatively longer PFS and OS than those treated with apatinib. Then, the multivariate analysis showed that compared with the second-line treatment, treated anlotinib or apatinib as third-line was an independent risk factor in PFS and OS, which meant that patients who received anlotinib or apatinib as second-line therapy had a better prognosis than those treated as third-line therapy. Although the baseline of treatment lines is inconsistent between patients in anlotinib and apatinib group, Table 1 has shown that the composition ratio of patients treated with second-line therapy in anlotinib group was significantly lower than that in apatinib group. It suggested that patients in apatinib group should have better survival than those in anlotinib group. However, in fact, treated with anlotinib was an independent protecting factor in OS. These results implied that the multi-targeted anti-angiogenic drug might have a better effect than single targeted anti-angiogenic drugs.

Similarly, the GOG-0227C study used bevacizumab as a single agent to treat recurrent and metastatic cervical cancer. $^{31}$ Among the enrolled patients, $73.9 \%$ were first-line drugs, and $26.1 \%$ were second-line drugs. ${ }^{31}$ The median PFS and OS of the study were 3.4 months and 7.3 months, respectively, which were lower than the results of this study. ${ }^{31}$ These results again support that anlotinib, a multi-targeted antiangiogenic drug, has better effects than a single antiangiogenic drug targeting vascular endothelial growth factors.

The common side effects in this study are similar to those of other anti-angiogenic drugs, including hand-foot syndrome, nausea, hypertension, fatigue, and neutropenia. High blood pressure, fatigue, and neutropenia had grade 3-4 adverse events, and their incidence was $10 \%, 5 \%$, and $5 \%$, respectively. RTOG0417 is a study to explore the efficacy and safety of bevacizumab combined with radiotherapy in advanced cervical cancer. ${ }^{32}$ The incidence of grade 3 and grade 4 bone marrow suppression in this study was $22.45 \%$ and $6.12 \%$, which were higher than ours. ${ }^{32}$ GOG240 is a study comparing the efficacy of bevacizumab combined with chemotherapy and chemotherapy alone. ${ }^{3} 6 \%$ of patients treated with bevacizumab combined with chemotherapy in GOG240 experienced grade 3 fistula adverse events, which was higher than that in the chemotherapy alone group. ${ }^{3}$ In this study, anlotinib did not have this adverse event regardless of single-agent, combined chemotherapy, combined radiotherapy. It can be seen that the adverse reactions of anlotinib in the treatment of cervical cancer are relatively low, and the side effects of the drug can be tolerated regardless of single-drug or combined use.

In conclusion, anlotinib has a certain effect in patients with persistent, recurrent, or metastatic cervical cancer who have failed first-line treatment, and the side effects are tolerable. The efficacy of anlotinib may be much better than the single anti-VEGF drugs. However, as the small sample size in this study and the self-limitation of retrospective study, further prospective clinical trials are needed to confirm our findings and establish the most suitable treatment strategy of anlotinib to benefit patients.

\section{Data Sharing Statement}

The raw data supporting the conclusions of this article will be made available by the authors without undue reservation. 


\section{Ethics Statement}

This study followed the principles of the Helsinki Declaration and was approved by the Ethics Committee of Zhongnan Hospital, Wuhan University, Hubei, China (Permit Number:2021030). All patients signed informed consent in this study.

\section{Funding}

This work was supported by Zhongnan Hospital of Wuhan University Science, Technology and Innovation Seed Fund (znpy2019078 and znpy2019080), the National Natural Science Foundation of China (81703037), and Leading Discipline in Oncology Fund of Hubei Province (XKJS202005).

\section{Disclosure}

The authors report no conflicts of interest in this work.

\section{References}

1. Sung H, Ferlay J, Siegel RL, et al. Global cancer statistics 2020: GLOBOCAN estimates of incidence and mortality worldwide for 36 cancers in 185 countries. CA Cancer J Clin. 2021;71(3):209-249. doi:10.3322/caac. 21660

2. Guitarte C, Alagkiozidis I, Mize B, Stevens E, Salame G, Lee YC. Glassy cell carcinoma of the cervix: a systematic review and meta-analysis. Gynecol Oncol. 2014;133(2):186-191. doi:10.1016/j. ygyno.2014.01.048

3. Tewari KS, Sill MW, Long HJ, et al. Improved survival with bevacizumab in advanced cervical cancer. $N$ Engl J Med. 2014;370 (8):734-743. doi:10.1056/NEJMoa1309748

4. Chuai Y, Rizzuto I, Zhang X, et al. Vascular endothelial growth factor (VEGF) targeting therapy for persistent, recurrent, or metastatic cervical cancer. Cochrane Database Syst Rev. 2021;3:CD013348.

5. Haibe Y, Kreidieh M, El Hajj H, et al. Resistance mechanisms to anti-angiogenic therapies in cancer. Front Oncol. 2020;10:221.

6. Syed YY. Anlotinib: first global approval. Drugs. 2018;78 (10):1057-1062. doi:10.1007/s40265-018-0939-x

7. Lin B, Song X, Yang D, Bai D, Yao Y, Lu N. Anlotinib inhibits angiogenesis via suppressing the activation of VEGFR2, PDGFR $\beta$ and FGFR1. Gene. 2018;654:77-86. doi:10.1016/j.gene.2018.02.026

8. Han B, Li K, Wang Q, et al. Effect of anlotinib as a third-line or further treatment on overall survival of patients with advanced non-small cell lung cancer: the ALTER 0303 phase 3 randomized clinical trial. JAMA Oncol. 2018;4(11):1569-1575. doi:10.1001/ jamaoncol.2018.3039

9. Song Y, Xiao J, Fang W, et al. The relationship between treatment-induced hypertension and efficacy of anlotinib in recurrent or metastatic esophageal squamous cell carcinoma. Cancer Biol Med. 2021;18(2):562-568. doi:10.20892/j.issn.2095-3941.2020.0187

10. Li S. Anlotinib: a novel targeted drug for bone and soft tissue sarcoma. Front Oncol. 2021;11:664853. doi:10.3389/fonc.2021.664853

11. Li D, Chi Y, Chen X, et al. Anlotinib in locally advanced or metastatic medullary thyroid carcinoma: a randomized, double-blind phase IIB trial. Clin Cancer Res. 2021;27(13):3567-3575. doi:10.1158/1078-0432.CCR-20-2950
12. Cui Q, Hu Y, Ma D, Liu H. A retrospective observational study of anlotinib in patients with platinum-resistant or platinum-refractory epithelial ovarian cancer. Drug Des Devel Ther. 2021;15:339-347. doi:10.2147/DDDT.S286529

13. Shen G, Zheng F, Ren D, et al. Anlotinib: a novel multi-targeting tyrosine kinase inhibitor in clinical development. J Hematol Oncol. 2018;11(1):120. doi:10.1186/s13045-018-0664-7

14. Yang H, Chen M, Mei Z, Xie C, Zhou Y, Qiu H. Effectiveness and prognostic factors of apatinib treatment in patients with recurrent or advanced cervical carcinoma: a retrospective study. Cancer Med. 2021;10(13):4282-4290. doi:10.1002/cam4.3966

15. Zhang L, Chen L, Yu H. Phase II study of apatinib, a novel tyrosine kinase inhibitor targeting tumor angiogenesis, as second-line treatment for recurrent or advanced cervical cancer patients. Invest New Drugs. 2020;38(4):1186-1191. doi:10.1007/s10637-019-00858-5

16. Yu J, Xu Z, Li A, et al. The efficacy and safety of apatinib treatment for patients with metastatic or recurrent cervical cancer: a retrospective study. Drug Des Devel Ther. 2019;13:3419-3424. doi:10.2147/DDDT.S214743

17. $\mathrm{Su} \mathrm{M}$, Gao Y, Ye X, et al. Clinical value of apatinib as a salvage treatment in patients with chemo-refractory advanced cervical cancer. Onco Targets Ther. 2019;12:9707-9713. doi:10.2147/OTT.S230406

18. Xiao Y, Cheng H, Wang L, Yu X. Clinical response and safety of apatinib monotherapy in recurrent, metastatic cervical cancer after failure of chemotherapy: a retrospective study. $J$ Gynecol Oncol. 2020;31(1):e2. doi:10.3802/jgo.2020.31.e2

19. Li N, Wang Z, Yuan G, et al. An oral small molecule VEGFR2 inhibitor, apatinib, in patients with recurrent or refractory cervical cancer: a real world study. $J$ Oncol. 2020;2020:3852373. doi: $10.1155 / 2020 / 3852373$

20. Lan C, Shen J, Wang Y, et al. Camrelizumab plus apatinib in patients with advanced cervical cancer (CLAP): a multicenter, open-label, single-arm, phase II trial. J Clin Oncol. 2020;38(34):4095-4106. doi:10.1200/JCO.20.01920

21. Folkman J, Merler E, Abernathy C, Williams G. Isolation of a tumor factor responsible for angiogenesis. $J$ Exp Med. 1971;133 (2):275-288. doi:10.1084/jem.133.2.275

22. van Beijnum JR, Nowak-Sliwinska P, Huijbers EJ, Thijssen VL, Griffioen AW. The great escape; the hallmarks of resistance to antiangiogenic therapy. Pharmacol Rev. 2015;67(2):441-461. doi:10.1124/pr.114.010215

23. Alldredge JK, Tewari KS. Clinical trials of antiangiogenesis therapy in recurrent/persistent and metastatic cervical cancer. Oncologist. 2016;21(5):576-585. doi:10.1634/theoncologist.2015-0393

24. Monk BJ, Mas Lopez L, Zarba JJ, et al. Phase II, open-label study of pazopanib or lapatinib monotherapy compared with pazopanib plus lapatinib combination therapy in patients with advanced and recurrent cervical cancer. J Clin Oncol. 2010;28(22):3562-3569. doi:10.1200/ JCO.2009.26.9571

25. Mackay HJ, Tinker A, Winquist E, et al. A phase II study of sunitinib in patients with locally advanced or metastatic cervical carcinoma: NCIC CTG Trial IND.184. Gynecol Oncol. 2010;116(2):163-167. doi:10.1016/j.ygyno.2009.08.012

26. Lu KV, Chang JP, Parachoniak CA, et al. VEGF inhibits tumor cell invasion and mesenchymal transition through a MET/VEGFR2 complex. Cancer Cell. 2012;22(1):21-35. doi:10.1016/j.ccr.2012.05.037

27. Moore DH, Blessing JA, McQuellon RP, et al. Phase III study of cisplatin with or without paclitaxel in stage IVB, recurrent, or persistent squamous cell carcinoma of the cervix: a gynecologic oncology group study. J Clin Oncol. 2004;22(15):3113-3119. doi:10.1200/JCO.2004.04.170

28. Xu Q, Chen C, Sun Y, et al. 865P A phase II study of sintilimab plus anlotinib for Chinese patients (pts) with persistent, recurrent, or metastatic cervical cancer (CC). Ann Oncol. 2020;31:S640. doi:10.1016/j.annonc.2020.08.1004 
29. Palakurthi S, Kuraguchi M, Zacharek SJ, et al. The combined effect of FGFR inhibition and PD-1 blockade promotes tumor-intrinsic induction of antitumor immunity. Cancer Immunol Res. 2019;7(9):1457-1471. doi:10.1158/2326-6066. CIR-18-0595

30. Liu S, Qin T, Liu Z, et al. Anlotinib alters tumor immune microenvironment by downregulating PD-L1 expression on vascular endothelial cells. Cell Death Dis. 2020;11(5):309. doi:10.1038/ s41419-020-2511-3

31. Monk BJ, Sill MW, Burger RA, Gray HJ, Buekers TE, Roman LD. Phase II trial of bevacizumab in the treatment of persistent or recurrent squamous cell carcinoma of the cervix: a gynecologic oncology group study. J Clin Oncol. 2009;27(7):1069-1074. doi:10.1200/ JCO.2008.18.9043
32. Schefter TE, Winter K, Kwon JS, et al. A phase II study of bevacizumab in combination with definitive radiotherapy and cisplatin chemotherapy in untreated patients with locally advanced cervical carcinoma: preliminary results of RTOG 0417. Int $J$ Radiat Oncol Biol Phys. 2012;83(4):1179-1184. doi:10.1016/j.ijrobp.2011.10.060

\section{Publish your work in this journal}

Drug Design, Development and Therapy is an international, peerreviewed open-access journal that spans the spectrum of drug design and development through to clinical applications. Clinical outcomes, patient safety, and programs for the development and effective, safe, and sustained use of medicines are a feature of the journal, which has also been accepted for indexing on PubMed Central. The manuscrip management system is completely online and includes a very quick and fair peer-review system, which is all easy to use. Visit http://www. dovepress.com/testimonials.php to read real quotes from published authors. 